УДК 621.039 .514

ЧИСЛЕННОЕ МОДЕЛИРОВАНИЕ ТЕПЛОМАССОПЕРЕНОСА В ПАРОГЕНЕРАТОРЕ РЕАКТОРНОЙ УСТАНОВКИ ТИПА БРЕСТ ПРИ ВОЗНИКНОВЕНИИ КРИСТАЛЛИЗАЦИИ СВИНЦОВОГО ТЕПЛОНОСИТЕЛЯ*

(C) 2018 г.

Чистов А.С. ${ }^{1}$, Савихин О.Г. ${ }^{2}$, Овчинников В.Ф. ${ }^{1}$, Кирюшина Е.В. ${ }^{1}$

${ }^{1}$ Научно-исследовательский институт механики Национального исследовательского Нижегородского государственного университета им. Н.И. Лобачевского, Нижний Новгород, Российская Федерация

${ }^{2}$ Национальный исследовательский Нижегородский государственный университет им. Н.И. Лобачевского, Нижний Новгород, Российская Федерация

chistov@mech.unn.ru

Поступила в редакияию 04.12.2017

Разработана методика численного моделирования нестационарного тепломассопереноса и теплопередачи в парогенераторе с жидкометаллическим теплоносителем при возникновении процесса кристаллизации. Методика учитывает различия протекания процесса в зависимости от состояния контактных поверхностей. На ее основе исследована динамика образования твердой фазы в свинцовом теплоносителе реакторной установки типа БРЕСТ в аварии с разгерметизацией паропровода парогенератора.

Расчеты выполнены с помощью программного комплекса РАСПЛАВ, который позволяет моделировать взаимосвязанную динамику теплогидравлических процессов в первом и втором контурах в одномерном равновесном приближении. Показано, что при отказе запорно-регулирующей арматуры по питательной воде происходит полное перекрытие проходного сечения аварийного парогенератора твердой фазой. В зависимости от состояния защитного оксидного слоя теплообменной поверхности затвердевание свинцового теплоносителя происходит либо на теплообменных трубах, либо в нижней части шахты парогенератора. В обоих случаях возникает угроза прекращения циркуляции в аварийной петле.

Дано краткое описание математической модели парогенератора и контура циркуляции установки в целом. Используется одномерное описание тепломассопереноса. Постулируется термодинамическое равновесие фаз на границе фазового перехода.

Приводятся результаты тестирования программного комплекса. Расчет разрыва трубопровода высокого давления протестирован на экспериментальных данных. Расчет динамики образования твердого слоя на поверхности теп-

* Выполнено при финансовой поддержке РФФИ (грант №16-08-01114 а). 
лообменных труб протестирован путем сравнения численного решения с аналитическим решением задачи Стефана.

Результаты исследования могут быть использованы при разработке проектов реакторов со свинцовым теплоносителем.

Ключевые слова: задача Стефана, запорный клапан, математическая модель, парогенератор, реакторная установка, свинцовый теплоноситель, смачиваемость поверхности, смешивающий подогреватель питательной воды, процесс кристаллизации, фазовый переход.

\section{Введение}

В настоящее время на площадке Сибирского химического комбината начата реализация проекта Росатома «Прорыв» [1], предусматривающего строительство опытно-демонстрационного реактора на быстрых нейтронах со свинцовым теплоносителем БРЕСТ-ОД-300 электрической мощностью 300 МВт и сопутствующего технологического комплекса замкнутого топливного цикла.

БРЕСТ-ОД-300 - реактор, в котором реализуется принцип естественной самозащищенности, с коэффициентом воспроизводства, большим единицы, с двухконтурной схемой отвода тепла и паротурбинным циклом преобразования энергии. Основные положения проекта изложены в [2-5].

При обосновании безопасности ядерных энергетических установок с тяжелыми жидкометаллическими теплоносителями [2, 6], имеющими высокую температуру плавления, можно выделить специфический класс аварий, в которых происходит образование твердой фазы в первом контуре парогенераторов (ПГ). Полное перекрытие проходного сечения одного или нескольких парогенераторов твердой фазой может привести к самым тяжелым последствиям: от разрыва теплообменных труб до полного прекращения циркуляции через реактор. Исследование этого класса аварий требует детального описания процесса затвердевания теплоносителя в парогенераторе.

В статье представлена методика численного моделирования динамики кристаллизации жидкометаллического теплоносителя в парогенераторе, основанная на математической модели [7]. Методика позволяет учитывать неопределенность, обусловленную состоянием защитного оксидного слоя на поверхности теплообменных труб, контактирующих с жидким металлом. Из [8, 9] следует, что несмачиваемость поверхности теплообмена металлом из-за наличия на ней оксидного слоя препятствует образованию центров кристаллизации. В этом случае либо кристаллические образования дрейфуют в турбулентном потоке в виде жидкометаллической суспензии, либо происходит переохлаждение потока. Накопление кристаллов в полости на выходе ПГ так же опасно, как и внезапное затвердевание переохлажденного расплава в этой полости. При отсутствии оксидного слоя на контактной поверхности затвердевание металла происходит на теплообменных трубах.

С использованием разработанной методики проведен расчетный анализ аварии с частичной разгерметизацией паропроводов одного из четырех ПГ реакторной установки типа БРЕСТ-ОД-300. Расчеты выполнены с помощью программного комплекса РАСПЛАВ $[10,11]$, который позволяет моделировать взаимосвязанную динамику теплогидравлических процессов в первом и втором контурах в одномерном равновесном приближении. Показано, что при несрабатывании отсечной арматуры по питательной воде происходит полное перекрытие проходного сечения первого 
контура аварийного ПГ твердой фазой свинца, вследствие чего возможно прекращение циркуляции в аварийной петле.

Приводятся результаты тестирования программного комплекса. Расчет разрыва трубопровода высокого давления протестирован на экспериментальных данных [12]. Расчет динамики образования твердого слоя на поверхности теплообменных труб протестирован путем сравнения численного решения с аналитическим решением задачи Стефана [13].

\section{1. Математическая модель}

Первый контур БРЕСТ-ОД-300 включает в себя реактор и четыре петли циркуляции теплоносителя, каждая из которых содержит один парогенератор, один главный циркуляционный насос (ГЦН) и тракты циркуляции теплоносителя. Второй контур представлен пароводяными трактами парогенераторов от смешивающего подогревателя питательной воды (СППВ) до турбины. Влияние остальной части второго контура учитывается через граничные условия. Моделирование динамики четырехпетлевого контура реакторной установки сводится к описанию двух петель: эквивалентной аварийной и эквивалентной неаварийной. Каждая из них моделирует динамику групп идентичных аварийных и идентичных неаварийных петель.

Расчетная блок-схема контура циркуляции реакторной установки типа БРЕСТ приведена на рис. 1.

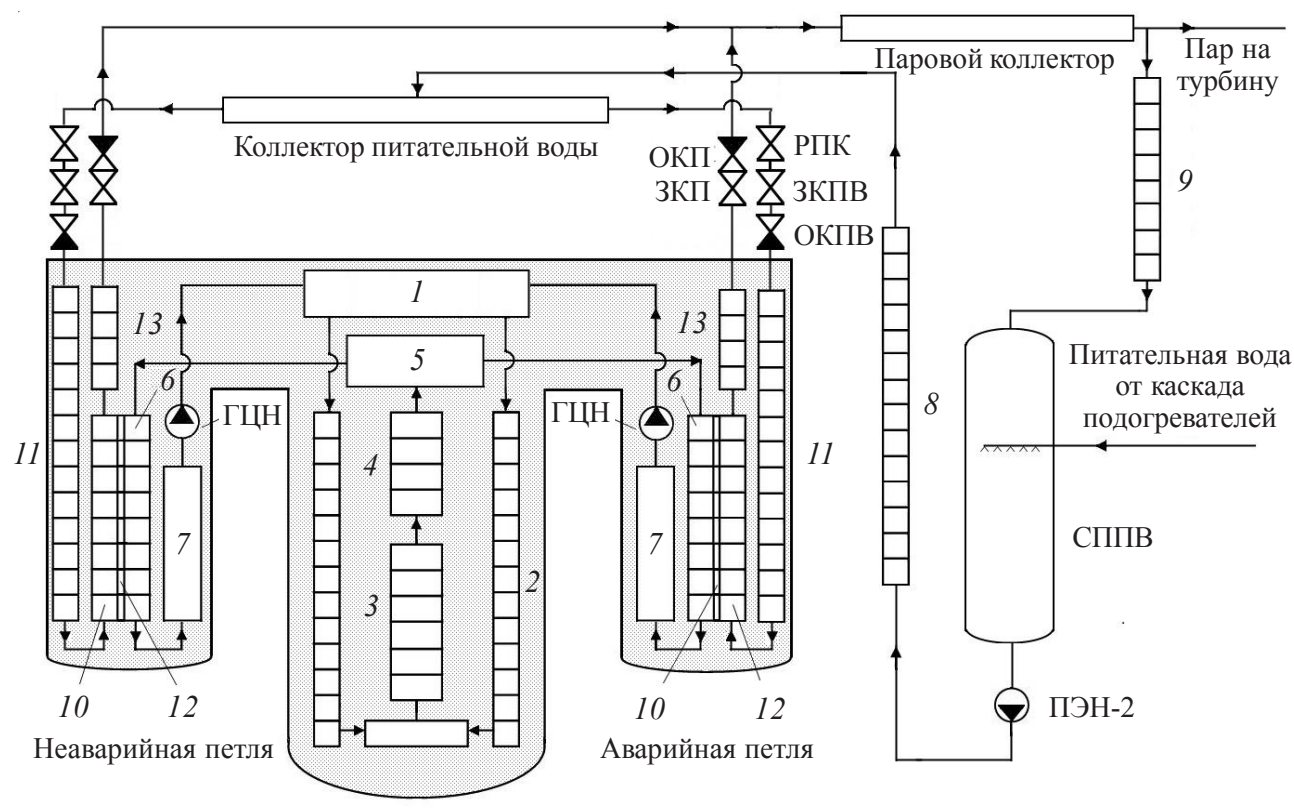

Рис. 1

Направления движения теплоносителей показаны стрелками, принцип циркуляции подробно описан в $[2,4]$. Первый и второй контуры представлены в виде набора взаимосвязанных теплогидравлических элементов (каналов): на схеме обозначены номерами 2-4, 6, 8, 9, 11-13. Последовательности каналов образуют тракты циркуляции теплоносителей. Тракты свинцового теплоносителя замыкаются на объемы со свободными уровнями: 1,5 - входная и выходная камеры реактора, 7 - вход- 
ная камера ГЦН. Свободные уровни объединены пространством, заполненным аргоном при атмосферном давлении $P_{a}$.

Парогенератор БРЕСТ-ОД-300 представляет собой теплообменник с противоточным движением сред $[2-4,14,15]$. Теплообменная поверхность 10 набрана из витых труб, внутри которых питательная вода нагревается до состояния перегретого пара. Трубная система ПГ имеет опускной подводящий участок 11 , змеевиковый участок активного теплообмена 12 и паровой участок 13. Змеевик 12 обогревается опускным потоком свинца в кольцевом пространстве между корпусом ПГ и внутренним цилиндрическим кожухом. Участок 11 расположен внутри кожуха.

Парогенераторы соединены с питательным и паровым коллекторами трубопроводами, на которых, согласно [4], установлены запорные клапаны по питательной воде (ЗКПВ) и по пару (ЗКП), а также регулирующие клапаны расхода питательной воды (РПК) и обратные клапаны по воде (ОКПВ) и по пару (ОКП).

Тепломассоперенос в каналах описан в одномерном гомогенном приближении. Алгоритмы решения конечно-разностной задачи для данной математической модели рассмотрены в [16]. Условиями сшивки на выходе предыдущего и входе последующего каналов являются равенства значений давления, удельной энтальпии и расхода.

В качестве начального условия при расчетах переходных процессов используется стационарный режим работы реакторной установки на номинальном уровне мощности. В качестве граничных условий используются давления на входе и выходе трактов циркуляции теплоносителей первого и второго контуров. Их значения составляют: по первому контуру $P_{11}=P_{a}+P_{i}, P_{21}=P_{a}+P_{j}$; по второму контуру $P_{12}=P_{0}(t)+H(t), P_{22}=P_{T}(t)$. Здесь $P_{i}, P_{j}$ - давления столбов уровней $i, j ; P_{0}(t)$, $P_{T}(t)$ - давления в СППВ и перед стопорно-регулирующим клапаном турбины; $H(t)-$ напор питательного электронасоса ПЭН-2.

Основные геометрические и режимные параметры, которые использовались в расчетах, представлены в таблице 1 и соответствуют данным из $[2,4,14,15]$.

\begin{tabular}{|l|c|c|c|c|c|c|}
\hline $\begin{array}{c}\text { Элемент } \\
\text { контура }\end{array}$ & Длина, м & $\begin{array}{c}\text { Площадь } \\
\text { сечения, } \mathrm{M}^{2}\end{array}$ & Объем, $\mathrm{M}^{3}$ & $\begin{array}{c}P_{\text {вх }} / \Delta P_{\text {тр, }} \\
\text { МПа }\end{array}$ & $\begin{array}{c}\text { Температура, } \\
{ }^{\circ} \mathrm{C}, \text { вх/вых }\end{array}$ & $\begin{array}{c}\text { Расход, } \\
\text { кг/c }\end{array}$ \\
\hline СППВ & - & - & 45 & $9 / 0,0$ & 340 & 420 \\
\hline 1-й контур ПГ & 7,0 & 1,0 & - & $0,1 / 0,07$ & $540 / 420$ & 10200 \\
\hline 2-й контур ПГ & 13,5 & 0,12 & - & $17,05 / 0,05$ & $340,5 / 506$ & 105 \\
\hline
\end{tabular}
[17].

Расчет стационарного режима ПГ производится по алгоритму, приведенному в

Тепловыделение в активной зоне рассчитывается в точечном приближении, остаточное тепловыделение - по формуле Унтермайера - Уэллса [18].

Разрыв паропровода моделируется уменьшением давления в сечении разрыва паропровода ПГ аварийной петли до атмосферного за время 0,5 c.

Подробнее рассмотрим методику расчета содержания твердой фазы в теплоносителе. Тепломассоперенос в первом контуре с учетом межфазного обмена через поверхность со свободной границей можно представить системой уравнений сохранения для однофазного потока с источником $J$ и переменной площадью сечения [7]: 


$$
\begin{aligned}
& \frac{\partial S_{1} \rho_{1}}{\partial t}+\frac{\partial G_{1}}{\partial z}=J, \\
& S_{1} \rho_{1} \frac{\partial i_{1}}{\partial t}+G_{1} \frac{\partial i_{1}}{\partial z}=q_{1}, \\
& \rho_{1}\left(\frac{\partial u_{1}}{\partial t}+u_{1} \frac{\partial u_{1}}{\partial z}\right)+\frac{\partial P}{\partial z}+\rho_{1} g_{z}+\frac{1}{2}\left(\frac{\xi_{1}}{d_{\Gamma 1}}+\xi_{0}\right) \rho_{1} u_{1}^{2}=0, \\
& J= \begin{cases}-\rho^{(2)} \Pi_{1}(\delta) \frac{\partial \delta}{\partial z}, & \delta>0, \\
0, & \delta=0,\end{cases} \\
& q_{1}= \begin{cases}\Pi_{1}(\delta) \alpha_{1}\left(T_{c r}-T_{1}\right), & \delta>0, \\
\Pi_{2} \alpha_{1}\left(T_{w}-T_{1}\right), & \delta=0,\end{cases} \\
& S_{1}= \begin{cases}S_{1}(\delta(t, z)), & \delta>0, \\
S_{1}^{*}, & \delta=0,\end{cases}
\end{aligned}
$$

а во втором контуре - системой уравнений гомогенной модели потока:

$$
\begin{gathered}
S_{2} \frac{\partial \rho_{2}}{\partial t}+\frac{\partial G_{2}}{\partial z_{2}}=0, \\
S_{2} \rho_{2} \frac{\partial i_{2}}{\partial t}+G_{2} \frac{\partial i_{2}}{\partial z_{2}}=q_{2}, \quad q_{2}=\alpha_{2} \Pi_{2}\left(T_{w}-T_{2}\right), \\
\frac{\partial G_{2}}{\partial t}+\frac{\partial}{\partial z_{2}}(G u)_{2}+\left(\frac{\xi G u}{2 d_{\Gamma}}\right)_{2}+S_{2} \frac{\partial P_{2}}{\partial z_{2}}+\left(S g_{z} \rho\right)_{2}=0,
\end{gathered}
$$

где $t$ - время; $z$ - продольная координата; $\rho$ - плотность; $G$ - массовый расход; $i-$ удельная энтальпия; $u$ - скорость; $q$ - тепловая мощность на единицу длины канала; $P$ - давление; $g_{z}$ - проекция ускорения свободного падения на направление движения; $S$ - площадь проходного сечения; $\xi$ - коэффициент гидравлического сопротивления трению; $\xi_{0}=\left(S_{1}^{*} / S_{1}(\delta)\right)^{2}\left(1+0,707 \sqrt{1-S_{1}(\delta) / S_{1}^{*}}\right)^{2}$ - коэффициент местного сопротивления при перекрытии выходного сечения ПГ твердой фазой [17]; $d_{\Gamma}-$ гидравлический диаметр; $\delta$ - толщина твердого слоя свинца; $T$ - температура; $\Pi_{1}(\delta)$, $\Pi_{2}-$ периметры поверхностей теплообмена со стороны первого и второго контуров. Нижние индексы обозначают: 1,2 - номер контура ПГ; $w$ - принадлежность к стенке трубы ПГ; верхний индекс (2) - принадлежность к твердой фазе; $c r$ - состояние кристаллизации-плавления. Процессы (1) и (2) связаны через процесс теплообмена в трубах ПГ, который с учетом образующегося твердого слоя можно описать согласно [7]:

$$
\begin{gathered}
\frac{\rho C}{\lambda} \frac{\partial T}{\partial t}=\frac{1}{r} \frac{\partial}{\partial r}\left(r \frac{\partial T}{\partial r}\right)+\frac{\partial^{2} T}{\partial z^{2}}, \quad r_{w}<r<r_{1}(t, z), r_{1}(t, z)=r_{w}+\delta(t, z), \\
q_{c r} \rho^{(2)} \frac{\partial \delta}{\partial t}=\alpha_{1}\left(T\left(r_{1}\right)-T_{1}\right)-\left.\lambda \frac{\partial T}{\partial n}\right|_{r=r_{1}}, \quad \alpha_{2}\left(T_{2}-T_{w}\right)-\left.\lambda \frac{\partial T}{\partial r}\right|_{r=r_{w}}=0,
\end{gathered}
$$

где $C, \lambda$ - соответственно удельная изобарная теплоемкость и коэффициент теплопроводности металла теплопередающего слоя; $r$ - радиальная координата; $r_{w}-$ pa- 
диус теплообменной трубы; $n$ - направление нормали к границе раздела фаз; $\alpha-$ коэффициенты теплоотдачи; $q_{c r}$ - скрытая теплота плавления.

Обозначив $l=L / 2, \delta_{l}=l-r_{w}, \delta^{*}=l / \cos (\pi / 6)-r_{w}$, определим $S_{1}=S_{1}(\delta(t, z))$ и $\Pi_{1}=\Pi_{1}(\delta(t, z))$ в (1) для трубной системы ПГ из $N$ теплообменных труб, расположенных в треугольной решетке с шагом $L$ :

$$
\begin{gathered}
S_{1}(\delta(t, z))= \begin{cases}S_{1}^{*}-\pi N\left(2 r_{w}+\delta\right) \delta, & r_{1}<l, \\
\frac{S_{1}\left(\delta_{l}\right)}{\sqrt{3}-\pi / 2}\left[\sqrt{3}+\left(3 \arccos \left(\frac{l}{r_{1}}\right)-\frac{\pi}{2}\right) \frac{r_{1}^{2}}{l^{2}}-\frac{3}{l} \sqrt{r_{1}^{2}-l^{2}}\right], & l \leq r_{1} \leq r_{w}+\delta^{*},\end{cases} \\
\Pi_{1}(\delta(t, z))=2 \pi\left(r_{w}+\delta\right) \cdot N \cdot K, \quad K= \begin{cases}1, & r_{1}<l, \\
1-\frac{6 \arccos \left(l / r_{1}\right)}{\pi}, & r_{1} \geq l .\end{cases}
\end{gathered}
$$

Условие на межфазной поверхности задачи (3) удобно выразить через подводимую в заданном сечении мощность

$$
\frac{\partial \delta}{\partial t}=\frac{q_{\Sigma}}{q_{c r} \rho^{(2)} \Pi_{1}}, \quad q_{\Sigma}=q_{1}-\left.\Pi_{1} \lambda \frac{\partial T}{\partial n}\right|_{r=r_{1}} .
$$

Численное интегрирование уравнения теплопроводности (3) осуществляется по явной разностной схеме с переменным числом узлов по пространственной координате.

В случае образования жидкометаллической суспензии объемное содержание твердой фазы определяется из соотношений осреднения гомогенной модели (см. [7]).

Функции состояния свинцового и пароводяного теплоносителей рассчитываются в виде $\rho=\rho(i, P), T=T(i, P)$ и аппроксимируются согласно $[19,20]$.

\section{2. Тестовые расчеты}

Тестирование компьютерной модели при расчете разрыва трубопровода высокого давления проведено с использованием экспериментальных данных [12]. На рис. 2 можно видеть приемлемое соответствие рассчитанного переходного процесса, вызванного резкой разгерметизацией трубы с водой под давлением $\sim 6,9$ МПа и температурой $242^{\circ} \mathrm{C}$, экспериментальным данным.

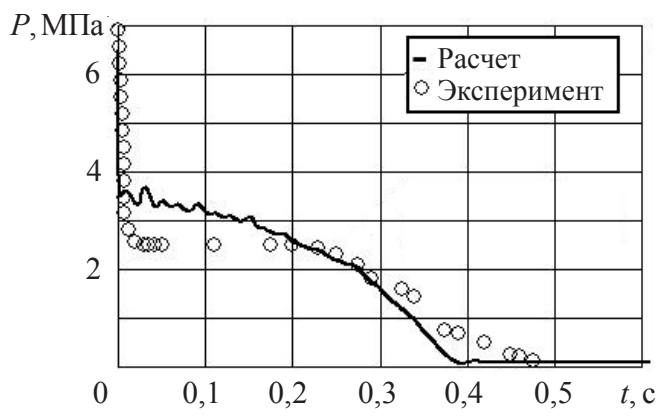

a)

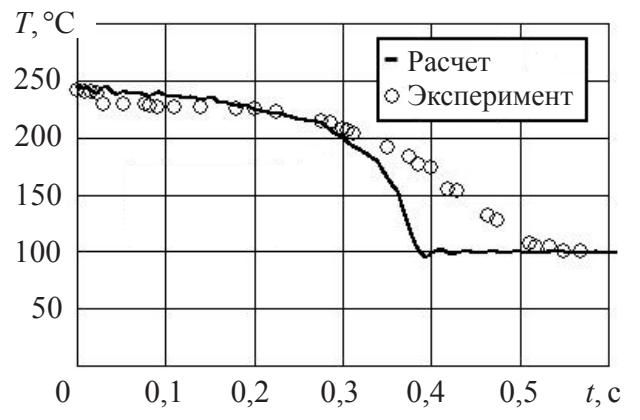

б)

Рис. 2 
На временном интервале, соответствующем истечению пароводяной смеси ( $P \approx$ const, $T \approx$ const), имеет место некоторое превышение рассчитанных значений давления и температуры по сравнению с экспериментальными значениями. При расчете аварии с разгерметизацией паропровода ПГ БРЕСТ-ОД-300 это обстоятельство позволит получить более консервативные оценки процесса образования твердой фазы в свинцовом теплоносителе.

Для тестирования модели, описывающей образование твердого слоя на трубах ПГ, рассмотрим квазистационарный процесс теплообмена, возникающий в изолированном ПГ в результате синхронного снижения температуры среды во втором контуре с номинальных значений до значения $220^{\circ} \mathrm{C}$ за 10 с (рис. 3).

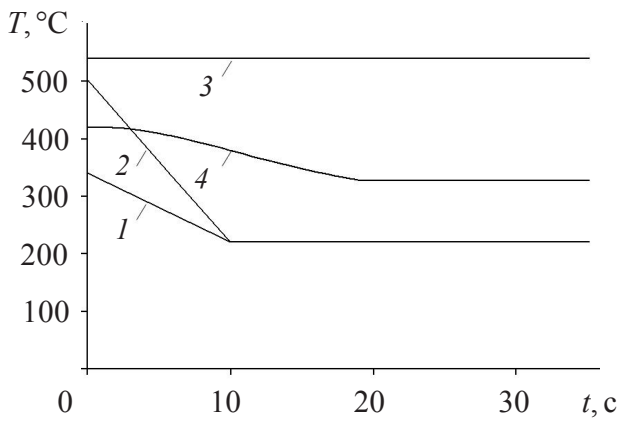

a)

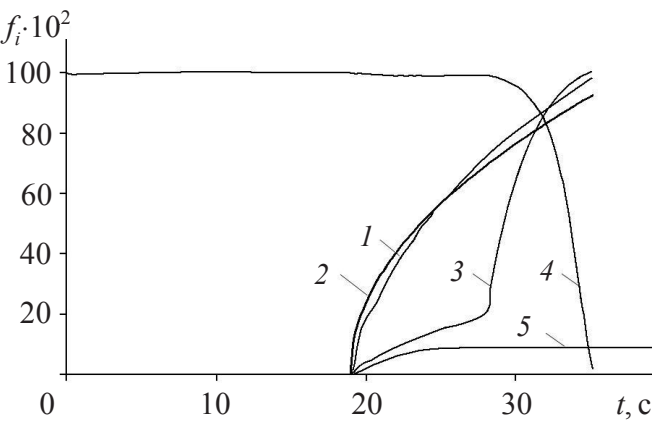

б)

Рис. 3

Линии 1, 2 на рис. $3 a$ показывают изменение температур во втором контуре, инициирующее переходный процесс. Температура жидкого свинца на входе первого контура остается постоянной (рис. $3 a$, линия 3 ), а на выходе достигает температуры кристаллизации $T_{c r}=327,5^{\circ} \mathrm{C}$ к 19 -й секунде (рис. $3 a$, кривая 4). С этого момента начинается образование твердого свинцового слоя на внешних поверхностях теплообменных труб. Кривые 1, 3 на рис. 36 показывают динамику нарастания твердого слоя и динамику перекрытия проходного сечения твердой фазой на выходе ПГ. В сужающемся сечении возникает дополнительное гидравлическое сопротивление потоку теплоносителя и, как следствие, уменьшение его расхода (рис. 36, кривая 4). Здесь и далее переменные $f_{i}, i>1$, нормируются на номинальные значения, а $f_{1}=$ $=\delta / \delta^{*}$ (см. (4)).

Задача Стефана о движении границы раздела фаз при стационарных граничных условиях имеет аналитическое решение $\delta(t)=A \sqrt{t-t_{0}}, A=$ const, $t_{0}-$ момент начала кристаллизации [13]. На участке ПГ, где идет процесс кристаллизации, на границах теплообменного слоя реализуются стационарные условия, и рассчитанная кривая 1 рис. 36 совпадает с аналитическим решением (рис. 36, кривая 2) с относительной погрешностью $\sim 5 \%$.

Кривые 3 и 5 на рис. 36 позволяют сопоставить динамику объемного содержания твердой фазы в переходном процессе в случае смачиваемой и несмачиваемой контактной поверхности расплавом.

\section{3. Авария с разгерметизацией паропровода парогенератора}

Рассматривается следующий сценарий аварии. За 0,5 с давление в сечении разрыва (перед ОКП) уменьшается до атмосферного. Расход пара в разрыв из осталь- 
ных ПГ отсекается обратным клапаном по пару. По причине провала давления во втором контуре закрывается стопорно-регулирующий клапан турбины и отключаются питательные насосы, производится сброс аварийной защиты реактора и отключение ГЦН. Далее происходит опорожнение СППВ через аварийный ПГ самотеком.

На рис. 4 показана динамика расходов и давлений во втором контуре. Расходы обозначены: 1 - в разрыв; 2, 5 - воды и пара в СППВ; 3 - на турбину; 4 - суммарный расход через неаварийные ПГ (см. рис. $4 a$ ). Давления обозначены: 6 - на выходе ПЭН-2; 7 - в СППВ; 8 - в сечении разрыва (см. рис. 4б).

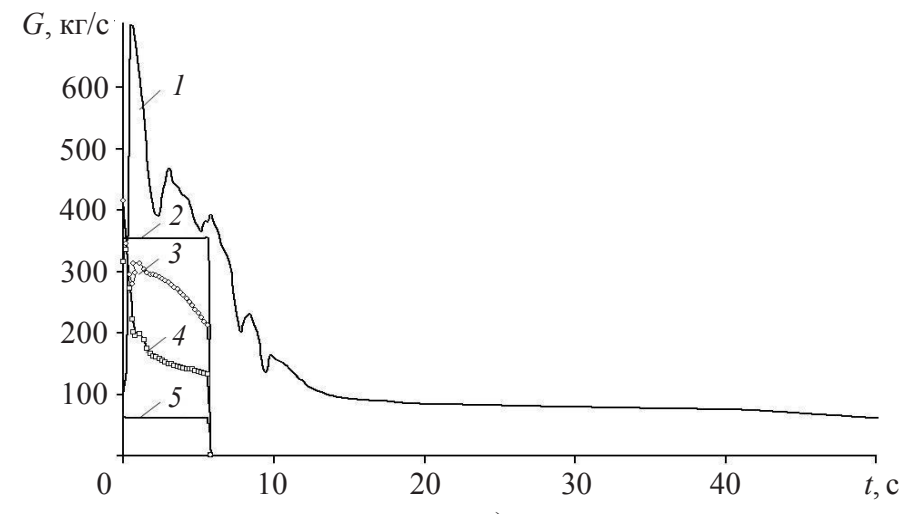

a)

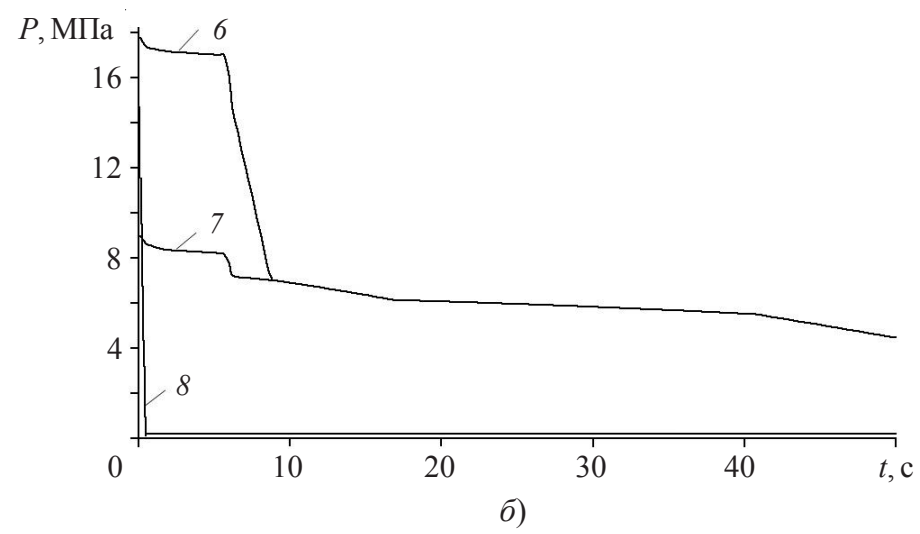

Рис. 4

Можно видеть, что во втором контуре ПГ через 20 с устанавливается квазистационарный режим с расходом, близким к номинальному, и давлением в СППВ $\sim 6$ МПа. К этому моменту температуры теплоносителей на входе первого и второго контуров достигают значений соответственно $\sim 75^{\circ} \mathrm{C}$ и $\sim 175^{\circ} \mathrm{C}$ (рис. $5 a$ ). Процесс кристаллизации в первом контуре начинается на 16-й секунде и зависит от состояния поверхности, контактирующей со свинцовым теплоносителем.

Динамика при отсутствии оксидного слоя на контактной поверхности. В этом случае поверхность приобретает свойство смачиваемости жидким металлом и образование твердого слоя происходит на теплообменных трубах.

На рис. 5а показана динамика температур на входе и выходе аварийного ПГ: 1 , 2 - первого контура; 3, 4- второго контура. Кривым на рис. 56 соответствуют следующие относительные величины: 1 - толщина твердого слоя на теплообменных 
трубах; 2 - перекрытие проходного сечения твердой фазой на выходе ПГ; 3 - расход свинцового теплоносителя. Кривая 2 имеет характерный разрыв производной при $t \approx 20$ с, связанный с соединением слоев затвердевшего свинца на соседних трубах. С этого момента процесс перекрытия сечения значительно ускоряется (см. (4), (5)). В момент $t \approx 26$ с происходит полное перекрытие проходного сечения и прекращение расхода свинцового теплоносителя в ПГ.
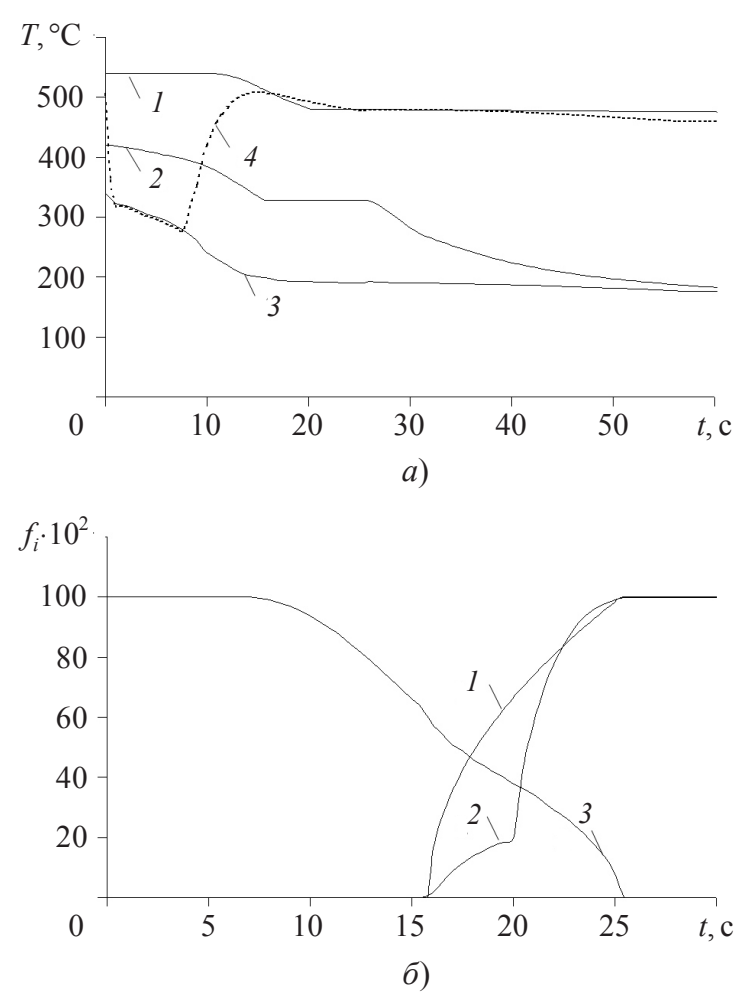

Рис. 5

При отсутствии конвективного теплоподвода процесс образования твердой фазы быстро распространяется по высоте теплообменного участка ПГ. На рис. 6 показана динамика распространения твердой фазы по высоте первого контура ПГ. Нормировка производится на максимальное значение $\delta^{*}$ (см. (4)). Можно видеть, что к моменту $t \approx 40$ с твердой фазой заполнена большая часть объема первого контура ПГ.

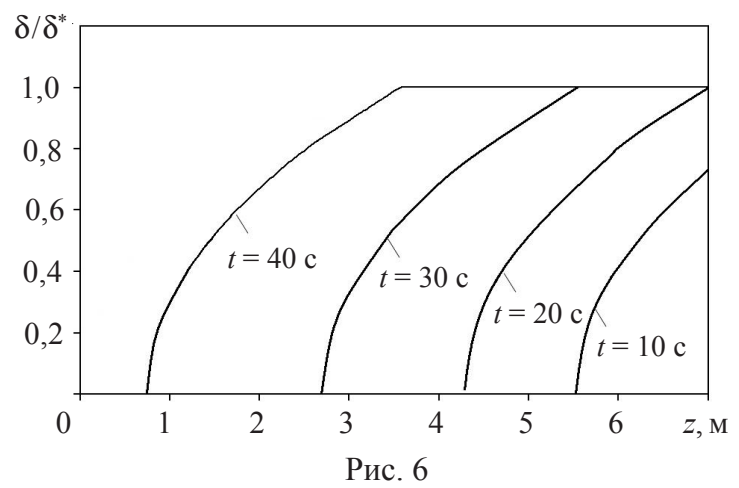


Динамика при наличии оксидного слоя на контактной поверхности. Оксидный слой обеспечивает несмачиваемость поверхности жидким металлом, что препятствует образованию на ней центров кристаллизации. Поток свинцового теплоносителя существует либо в равновесном двухфазном состоянии в виде жидкометаллической суспензии, либо в переохлажденном состоянии. На рис. 7 показан переходный процесс при выносе твердой фазы из ПГ и накоплении ее в нижней части входной камеры ГЦН. Нумерация кривых на рис. $7 a$ такая же, как на рис. $5 a$.
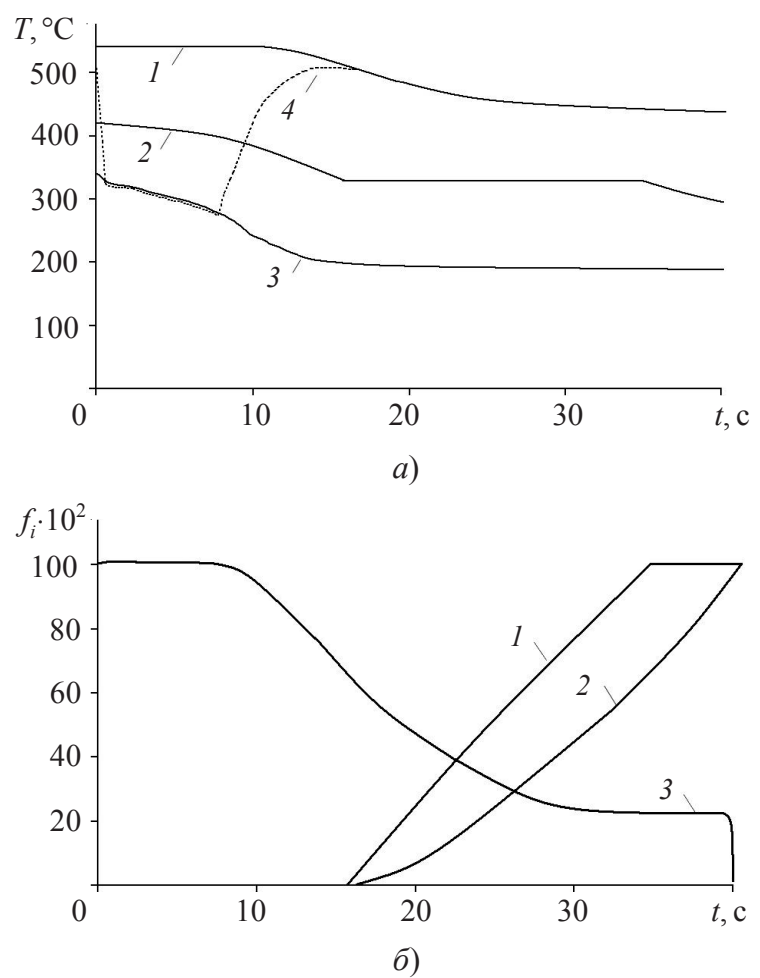

Рис. 7

Сравнивая переходные процессы в аварийном ПГ при наличии (см. рис. 7) и отсутствии (см. рис. 5) оксидного слоя на контактной поверхности, можно видеть, что затвердевание свинца на стенке приводит к более быстрому перекрытию сечения на выходе ПГ. Это связано с уменьшением расхода, вызванным ростом гидравлического сопротивления в сужающемся потоке. Кривая 2 на рис. 76 показывает динамику накопления твердой фазы в полусферической полости на выходе ПГ:

$$
\bar{V}=\frac{1}{V} \int_{t_{0}}^{t} \frac{\varphi^{(2)} G_{1}}{\rho_{1}} d t,
$$

где $\varphi^{(2)}$ - объемное содержание твердой фазы на выходе ПГ (рис. 7б, кривая 1 ); $V-$ объем полости непосредственно под корпусом ПГ. При заполнении всего объема твердой фазой $(\bar{V}=100 \%)$ выходное сечение первого контура ПГ перекрывается, и циркуляция свинца в аварийной петле прекращается (рис. 7б, кривая 3).

Представленные модели оценивают динамику образования твердой фазы в двух качественно различных случаях. На практике может реализоваться некоторый промежуточный вариант, сочетающий образование твердого слоя на трубах ПГ с затвердеванием теплоносителя в объеме на выходе ПГ. 


\section{Выводы}

Разработана методика численного моделирования динамики процесса кристаллизации в парогенераторе с жидкометаллическим теплоносителем, которая позволяет учитывать неопределенность, связанную с состоянием контактной поверхности. На ее основе проведено численное исследование процесса кристаллизации свинцового теплоносителя в парогенераторе реакторной установки типа БРЕСТ-ОД-300, возникающего в результате разгерметизации выходного паропровода.

При несрабатывании запорно-регулирующей арматуры по питательной воде происходит полное перекрытие проходного сечения аварийного парогенератора твердой фазой. В зависимости от состояния защитного оксидного слоя теплообменной поверхности затвердевание свинцового теплоносителя происходит либо на теплообменных трубах, либо в нижней части шахты парогенератора. В обоих случаях возникает угроза прекращения циркуляции в аварийной петле, что требует дальнейшего детального исследования аварий данного класса.

\section{Список литературы}

1. Проект «Прорыв» остается приоритетным для Росатома [Электронный ресурс]. Режим доступа: http: //www.rosatom.ru/journalist/news/proekt-proryv-ostaetsya-prioritetnym-dlyarosatoma (Дата обращения: 12.09.2017).

2. Драгунов Ю.Г., Лемехов В.В., Смирнов В.С., Чернецов Н.Г. Технические решения и этапы разработки реакторной установки БРЕСТ-ОД-300. Атомная энергия. 2012. Т. 113. Вып. 1. С. 58-65.

3. Филин А.И., Цикунов В.С., Попов С.В., Нестеров Ю.В., Шварц А.Л., Колбасников А.В., Шмуклер Б.И., Гомболевский В.И., Радин Ю.А., Вахрушин М.П. Разработка схем, оборудования и режимов работы второго контура перспективной АЭС. Теплоэнергетика. 2001. №6. C. 27-31.

4. Нестеров Ю.В., Лисянский А.С., Макарова Е.И., Бальва Л.Я., Приходько П.Ю. Тепловая схема и оборудование II контура энергоблока АЭС с реакторной установкой БРЕСТОД-300. Теплоэнергетика. 2011. №6. С. 32-36.

5. Дидорин Д.В., Когут В.А., Муратов А.Г., Тюков В.В., Моисеев А.В. Анализ безопасности РУ БРЕСТ-ОД-300 при нарушении условий нормальной эксплуатации. Инновационные проекты и технологии ядерной энергетики: Труды Междунар. научно-техн. конф. ОАО «НИКИЭТ», Москва. 7-10 октября 2014 г. [Электронный ресурс]. Режим доступа: http:// www.nikiet.ru/images/stories/NIKIET/Publications/Conf/mntk_nikiet_2014/I-3_rus.pdf (Дата обращения: 12.09.2017).

6. Alemberti A. European lead fast reactor - ELSY. Nuclear Engineering and Design. 2011. Vol. 241. Iss. 9. P. 3470-3480.

7. Чистов А.С., Савихин О.Г., Овчинников В.Ф., Николаев М.Я. Математическая модель нестационарного тепломассопереноса в канале с жидкометаллическим теплоносителем с учетом процесса кристаллизации-плавления. Проблемы прочности и пластичности. 2016. T. 78. №4. С. 368-377.

8. Безносов А.В., Молодцов А.А., Назаров А.В., Савинов С.Ю., Кудрин О.О. Исследование теплоотдачи от свинцового теплоносителя к продольнообтекаемой трубе. Теплофизика и аэромеханика. 2007. Т. 14. №3. С. 429-436.

9. Махов К.А. Исследование структуры и триботехнических характеристик пристенного слоя в потоке ТЖМТ применительно к элементам контуров с реакторами на быстрых нейтронах, охлаждаемыми ТЖМТ: Дис. ... канд. техн. наук. Н. Новгород. НГТУ им. Р.Е. Алексеева, 2015. 164 с.

10. Будников В.И., Савихин О.Г., Чистов А.С. Расчетное исследование динамики замораживания свинцового теплоносителя при разрыве паропровода РУ с реактором БРЕСТ- 
ОД-300. Научная сессия МИФИ-2002. Научно-инновационное сотрудничество: Сборник трудов научно-техн. конф. Ч. 2. М.: МИФИ, 2002. С. 164-165.

11. Будников В.И., Савихин О.Г., Чистов А.С. Расчетный анализ проблем безопасности, возникающих при разгерметизации контуров циркуляции в РУ с реакторами типа ВВЭР и БРЕСТ. Нижегородский университет им. Н.И. Лобачевского. Важнейшие научные резульmaты. Нижний Новгород: Изд-во ННГУ, 2004. С. 63-64.

12. Edwards A.R., O'Brien F.P. Studies of phenomena connected with the depressurization of water reactors. Journal of the British Nuclear Energy Society. 1970. Vol. 9. P. 125-135.

13. Мейрманов А.М. Задача Стефана. Новосибирск: Наука, 1986. 239 с.

14. БРЕСТ: быстрый реактор со свиниовым теплоносителем и пристанционным топливнылм циклом [Электронный ресурс]. Режим доступа: http://www.atomic-energy.ru/technology/ 36000. (Дата обращения: 12.09.2017).

15. Семченков А.А., Чеков М.Е., Васильев С.В., Кузьминов Ю.В. Парогенератор РУ БРЕСТ-ОД-300: расчетно-экспериментальное обоснование. Вопросы атомной науки и техники. Серия: Обеспечение безопасности АЭС. 2016. №36. С. 33-37.

16. Будников В.И., Савихин О.Г., Чистов А.С. Численное моделирование нестационарных теплогидравлических процессов в контурах циркуляции водяного теплоносителя перспективной АЭС. Вестник Нижегородского государственного университета им. Н.И. Лобачевского. 2013. №1 (1). С. 158-163.

17. Кириллов П.Л., Юрьев Ю.С., Бобков В.П. Справочник по теплогидравлическим расчетам (ядерные реакторы, теплообменники, парогенераторы). М.: Энергоатомиздат, 1990. $359 \mathrm{c}$.

18. Овчинников Ф.Я., Семенов В.В. Эксплуатационные режимы водо-водяных энергетических реакторов. М.: Энергоатомиздат, 1988. 359 с.

19. Iida T., Guthrie R.I.L. The Physical Properties of Liquid Metals. Oxford, UK: Clarendon Press, 1988. 285 p.

20. Савихин О.Г., Линник С.В., Савихин А.О. Аппроксимация производных термодинамических функций для воды, пара и пароводяной смеси. Вестник Нижегородского государственного университета им. Н.И. Лобачевского. 2011. №4 (3). С. 1090-1091.

\section{References}

1. Proekt "Proryv" ostaetsya prioritetnym dlya Rosatoma [The "PRORYV "Project Remains a Priority for Rosatom] Electronic resource. Access mode: http: //www.rosatom.ru/journalist/news/ proekt-proryv-ostaetsya-prioritetnym-dlya-rosatoma (Date of application: 12.09.2017) (In Russian).

2. Dragunov Yu.G., Lemekhov V.V., Smirnov V.S., Chernetsov N.G. Technical solutions and development stages for the BREST-OD-300 reactor unit. Atomic Energy. 2012. Vol. 113. Iss. 1. P. 70-77. DOI: 10.1007/s10512-012-9597-3.

3. Filin A.I., Tsikunov V.S., Popov S.V., Nesterov Yu.V., Shvarts A.L., Kolbasnikov A.V., Shmukler B.I., Gombolevskii V.I., Radin Yu.A., Vakhrushin M.P. Development of the thermal schemes, equipment and operating regimes of the second circuit of a promising nuclear power station. Thermal Engineering. 2001. No 6. P. 458-463.

4. Nesterov Y.V., Lisyanskii A.S., Makarova E.I., Bal'va L.Ya., Prikhod'ko P.Yu. The thermal process diagram and equipment of the secondary coolant circuit of a nuclear power station unit based on the BREST-OD-300 reactor installation for subcritical steam conditions. Thermal Engineering. 2011. Vol. 58. Iss. 6. P. 438-482. DOI: 10.1134/S0040601511060103.

5. Didorin D.V., Kogut V.A., Muratov A.G., Tyukov V.V., Moiseev A.V. Analysis of BRESTOD-300 safety during anticipated operational occurrences. Innovative Projects and Technologies of Nuclear Power: Digest of International Scientific and Technical Conference. Moscow. October 7-10, 2014. Electronic resource. Access mode: http://www.nikiet.ru/images/stories/NIKIET/ Publications/Conf/mntk nikiet 2014/I-3 rus.pdf (Date of application: 12.09.2017) (In Russian).

6. Alemberti A. European lead fast reactor - ELSY. Nuclear Engineering and Design. 2011. Vol. 241. Iss. 9. P. 3470-3480.

7. Chistov A.S., Savikhin O.G., Ovchinnikov V.F., Nikolaev M.Ya. Matematicheskaya model nestatsionarnogo teplomassoperenosa $\mathrm{v}$ kanale s zhidkometallicheskim teplonositelem s uchetom 
protsessa kristallizatsii-plavleniya [Mathematical model of non-stationary heat and mass transfer in a channel with liquid-metal coolant taking into account the crystallization-melting process]. Problemy prochnosti i plastichnosti [Problems of Strength and Plasticity]. 2016. Vol. 78. No 4. P. 368-377 (In Russian).

8. Beznosov A.V., Molodtsov A.A., Nazarov A.V., Savinov S.Y., Kudrin O.O. Investigation of heat transfer from a lead heat carrier to a tube streamlined longitudinally. Thermophysics and Aeromechanics. 2007. Vol. 14. No 3. P. 411-418.

9. Makhov K.A. Issledovanie struktury i tribotekhnicheskikh kharakteristik pristennogo sloya $\mathrm{v}$ potoke TZhMT primenitel'no k elementam konturov s reaktorami na bystrykh neytronakh, okhlazhdaemymi TZhMT [The study of the structure and tribotechnical characteristics of boundarylayer flow HLMC in relation to the elements of the circuits with fast neutron reactors cooled HLMC]. Dis... kand. tekhn. nauk [Cand. Sci. (Engineering). Dissertation]. Nizhni Novgorod. NNSTU Publ. 2015. 164 p. (In Russian).

10. Budnikov V.I., Savikhin O.G., Chistov A.S. Raschetnoe issledovanie dinamiki zamorazhivaniya svintsovogo teplonositelya pri razryve paroprovoda RU s reaktorom BREST-OD-300 [Numerical study of the lead coolant freezing dynamics at break of steam line of NPP with BRESTOD-300 reactor]. Nauchnaya sessiya MIFI-2002. Nauchno-innovatsionnoe sotrudnichestvo: Sbornik trudov nauchno-tekhnicheskoy konferentsii. Ch. 2. [Scientific and Technical Conference "Scientific-innovative Cooperation". Collection of Proceedinds]. Pt. 2. Moscow. MIFI Publ. 2002. P. 164-165 (In Russian).

11. Budnikov V.I., Savikhin O.G., Chistov A.S. Raschetnyy analiz problem bezopasnosti, voznikayushchikh pri razgermetizatsii konturov tsirkulyatsii v RU s reaktorami tipa VVER i BREST [Computational analysis of security problems that occur when depressurization of nuclear power plan circuits with VVER and BREST type reactors]. Nizhegorodskiy universitet im. N.I. Lobachevskogo. Vazhneyshie nauchnye rezultaty [Lobachevsky State University of Nizhni Novgorod. The Most Important Scientific Results]. Nizhni Novgorod. UNN Publ. 2004. P. 63-64 (In Russian).

12. Edwards A.R., O'Brien F.P. Studies of phenomena connected with the depressurization of water reactors. Journal of the British Nuclear Energy Society. 1970. Vol. 9. P. 125-135.

13. Meyrmanov A.M. Zadacha Stefana [The Stefan Problem]. Novosibirsk. Nauka Publ. 1986. 239 p. (In Russian).

14. BREST: bystryy reaktor so svintsovym teplonositelem i pristantsionnym toplivnym tsiklom [BREST: Fast Reactor with Lead Coolant and On-site Fuel Cycle]. Electronic resource. Access mode: http://www.atomic-energy.ru/technology/36000 (Date of application: 12.09.2017) (In Russian).

15. Semchenkov A.A., Chekov M.E., Vasilyev S.V., Kuzminov Y.V. Parogenerator RU BRESTOD-300: raschetno-eksperimentalnoe obosnovanie [Steam generator RU BREST-OD-300: calculation and experimental justification]. Voprosy atomnoy nauki i tekhniki. Seriya: Obespechenie bezopasnosti AES. [Questions of Atomic Science and Technology. Series: Ensuring the Safety of Nuclear Power Plants]. 2016. No 36. P. 33-37 (In Russian).

16. Budnikov V.I., Savikhin O.G., Chistov A.S. Chislennoe modelirovanie nestatsionarnykh teplogidravlicheskikh protsessov v konturakh tsirkulyatsii vodyanogo teplonositelya perspektivnoy AES [Numerical simulation of non-stationary thermal-hydraulic processes in the water coolant circulation loops of an advanced nuclear power plant]. Vestnik Nizhegorodskogo gosudarstvennogo universiteta im. N.I. Lobachevskogo [Bulletin of UNN]. 2013. No 1(1). P. 158-163 (In Russian).

17. Kirillov P.L., Yur'yev Yu.S., Bobkov V.P. Spravochnik po teplogidravlicheskim raschetam (yadernye reaktory, teploobmenniki, parogeneratory) $[A$ Handbook on Thermohydraulic Calculations (Nuclear Reactors, Heat Exchangers, Steam Generators)]. Moscow. Energoatomizdat Publ. 1990. 359 p. (In Russian).

18. Ovchinnikov F.Y., Semenov V.V. Ekspluatatsionnye rezhimy vodo-vodyanykh energeticheskikh reaktorov [Operational Modes of Water-Water Power Reactors]. Moscow. Energoatomizdat Publ. 1988. 359 p. (In Russian).

19. Iida T., Guthrie R.I.L. The Physical Properties of Liquid Metals. Oxford. UK. Clarendon Press. 1988. 285 p.

20. Savikhin O.G., Linnik S.V., Savikhin A.O. Approksimatsiya proizvodnykh termodinamicheskikh funktsiy dlya vody, para i parovodyanoy smesi [Approximation of derivatives of thermo- 
dynamic functions for water, steam and mixture]. Vestnik Nizhegorodskogo gosudarstvennogo universiteta im. N.I. Lobachevskogo [Bulletin of UNN]. 2011. No 4 (3). P. 1090-1091 (In Russian).

\title{
NUMERICAL SIMULATION OF HEAT AND MASS TRANSFER IN THE STEAM GENERATOR OF THE BREST TYPE REACTOR PLANT IN THE EVENT OF CRYSTALLIZATION OF LEAD COOLANT
}

\author{
Chistov A.S. ${ }^{1}$, Savikhin O.G. ${ }^{2}$, Ovchinnikov V.F. ${ }^{1}$, Kiryushina E.V. ${ }^{1}$ \\ ${ }^{1}$ Research Institute of Mechanics University of Nizhni Novgorod, \\ Nizhni Novgorod, Russian Federation \\ ${ }^{2}$ Lobachevsky State University of Nizhni Novgorod, \\ Nizhni Novgorod, Russian Federation
}

The method of numerical simulation has been developed describing non-stationary heat and mass transfer and thermal conductivity in a steam generator with liquid metal coolant when there is a crystallization process. The method takes into account the differences of the process depending on the condition of the contact surfaces.

On its basis the dynamics of solid phase formation in a lead-coolant of reactor plant BREST type during the accident with the steam generator steam line depressurization has been studied. The calculations are carried out with using of the RASPLAV software complex, which allows modeling the interconnected dynamics of thermal-hydraulic processes in the first and second circuits in terms of the one-dimensional equilibrium approximation. It is shown that at failure of feed water lock-regulatory armature there is a full closure of flow section of the emergency steam generator first circuit by solid phase. Depending on the condition of the protective oxide layer of the heat exchange surface the solidification of lead coolant takes place either on the heat exchanger tubes or in the lower part of the steam generator reservoir. In both cases, there is a threat of cessation of circulation in the emergency loop.

A brief description of the mathematical model of the steam generator and the reactor plant circuit as a whole is given. A one-dimensional description of heat and mass transfer is used. A thermodynamic equilibrium of the phases at the boundary of phase transfer is postulated.

The test results of the programmed numerical model are given. The calculation of the high pressure pipeline rupture is tested by comparison with the experimental data. The calculation of the solid layer formation dynamics on the surface of heat exchange tubes is tested by comparing the numerical solution with the analytical solution of the Stefan problem.

The results of this research can be used for design of reactors with a lead coolant.

Keywords: Stefan problem, stop valve, mathematical model, steam generator, reactor plant, lead coolant, surface wettability, mixing feedwater heater, crystallization process, phase transfer. 